\title{
Formação de redes de cooperação para o desenvolvimento de e-marketplaces verticais
}

\author{
Marcio Toyoki Morinishi ${ }^{\mathrm{a}, *}$, Fábio Müller Guerrini ${ }^{\mathrm{b}}$ \\ a,*toyoki@gmail.com, USP, Brasil \\ bguerrini@sc.usp.br, USP, Brasil
}

\begin{abstract}
Resumo
Atualmente, os projetos de e-marketplaces verticais têm sido coordenados por empresas de um mesmo setor econômico, às vezes concorrentes, que se reúnem com o objetivo de aumentar a competitividade do setor. Dentro desse contexto, este artigo analisa os fatores determinantes para cooperação no desenvolvimento dos e-marketplaces e quais os seus efeitos sobre as assimetrias de informação entre compradores e vendedores. É apresentado um estudo de caso no setor da construção civil, no qual construtoras brasileiras têm como proposta compartilhar custos e riscos para o desenvolvimento de um e-marketplace.
\end{abstract}

Palavras-chave

Redes de cooperação. Comércio eletrônico. Assimetria de informação. Construção civil.

\section{Introdução}

A intensa concorrência vem impondo a elevação dos níveis de exigência da demanda. 0 aumento na qualidade dos produtos e serviços, concomitantemente à necessidade de redução dos custos, tornou-se requisito para as empresas manterem a competitividade.

Para enfrentar as instabilidades, as empresas são impelidas pelo mercado a possuir capacidades em termos de recursos e atividades que elas somente conseguiriam obter por meio da cooperação (GEBREKIDAN; AWUAH, 2002). A cooperação entre as empresas permite que elas tenham acesso à informação, recursos, mercados e tecnologias, com vantagens de aumentar seus conhecimentos, obter economias de escala e escopo, compartilhar riscos e combinar competências com outras empresas, tornando-as mais aptas a explorar novas oportunidades do mercado (GULATI; NOHRIA; ZAHEER, 2000).

Atualmente, há a cooperação entre empresas, muitas vezes concorrentes, para o desenvolvimento de plataformas tecnológicas. Esses projetos, normalmente coordenados por uma associação da indústria ou incorporados na estrutura de consórcio, buscam compartilhar ideias, tecnologias e vantagens entre seus membros (ALBERTIN, 2004).
Um exemplo é o Covisint - grupo formado pela DaimlerChrysler, Ford, General Motors, Renault, Nissan e Peugeot-Citroën -, cujo objetivo é combinar esforços para integrar montadoras e fornecedores e aumentar a eficiência da cadeia de suprimentos no setor automobilistico, através de um e-marketplace vertical (KANDAMPULLY, 2003).

0 e-marketplace (mercado eletrônico) provê a infraestrutura tecnológica para criação de um local virtual onde compradores e fornecedores realizam transações comerciais através da internet (BAKOS, 1998). 0 termo vertical indica que o e-marketplaceé destinado a um determinado segmento da indústria (KAPLAN; SAWHNEY, 2000).

Ao atuar como intermediário eletrônico, o e-marketplace beneficia tanto fornecedores quanto compradores. Para os fornecedores, propicia um meio eficiente para publicidade e redução dos custos de suas operações comerciais e financeiras. Para os compradores diminui o tempo e os custos do processo de seleção de fornecedores, aumenta o número das alternativas a serem consideradas, permite a customização dos produtos oferecidos, facilita a obtenção de informações sobre os produtos e permite a eliminação dos intermediários 
tradicionais da cadeia produtiva (BAKOS, 1998; WIGAND; BENJAMIN, 1995).

Sob a perspectiva da teoria dos custos de transação, os e-marketplaces auxiliam na redução dos custos de coordenação com o mercado, pois auxiliam as empresas na procura por fornecedores, na negociação dos preços e no gerenciamento dos contratos (MALONE; YATES; BENJAMIN, 1997). Ainda assim, é necessário analisar o papel dos e-marketplaces sobre os riscos da transação, visto que estes também influenciam seus custos. Os riscos da transação referem-se aos custos relacionados com a possibilidade de o relacionamento ser explorado através do oportunismo (CLEMONS; ROW, 1992).

0 comportamento oportunista existe quando um dos agentes atua estrategicamente, tentando concretizar seus próprios interesses em detrimento dos interesses de outros agentes e sob desobediência das normas sociais (WILLIAMSON, 1985). A suposição do oportunismo numa situação em que há assimetrias de informação, isto é, casos em que pessoas ou empresas têm diferentes níveis de conhecimento sobre as circunstâncias de uma negociação, pode aumentar os custos ou diminuir os benefícios da outra parte, que é incapaz de monitorar ou controlar as ações do agente oportunista (BYRNS; STONE, 1996).

A literatura oferece algumas formas de minimizar as assimetrias de informação, sendo que alguns autores sugerem a utilização de sistemas de informação para diminuir o comportamento oportunista dos agentes (EISENHARDT, 1989; CLEMONS; ROW, 1992).

Dentro desse contexto, o artigo analisa 0 desenvolvimento do e-marketplacesob a perspectiva das redes de cooperação e das assimetrias de informação. Para tanto, foi realizado um estudo de caso no setor da construção civil, com foco na análise dos procedimentos comuns entre as construtoras e os requisitos do e-marketplace demandados para 0 processo de aquisição de produtos e serviços.

Adicionalmente, foram desenvolvidos modelos que serviram como guia de referência para as construtoras. Para a modelagem do projeto, utilizou-se o EKD (Enterprise Knowledge Development), visto que seus modelos representam de forma estruturada a relação entre os objetivos, os processos de negócio e os sistemas de informação, independentemente da plataforma tecnológica adotada (BUBENKO; PERSSON; STIRNA, 1998).

\section{Redes de cooperação entre empresas}

A teoria do custo de transação, idealizada por Coase (1937) e posteriormente desenvolvida por
Williamson (1985), foi uma evolução em relação às teorias econômicas neoclássicas ao reconhecer que, além dos custos de produção, existem custos associados ao emprego de recursos para coordenação das atividades econômicas com 0 "mercado".

Os custos de produção são as despesas inerentes ao processo de criação e distribuição de um bem ou recurso que está sendo produzido. Por outro lado, a empresa ao recorrer ao "mercado" para contratação de fornecedores incorre em custos de transação que incluem: custos de contato (exemplo: procurar informação), custos de contrato (exemplo: negociação e formulação de contratos), custos de monitoramento (exemplo: verificação da qualidade, quantidade, preços e prazos) e custos de adaptação (exemplo: mudanças durante a validade do contrato) (WILLIAMSON, 1981; WIGAND; PICOT; REICHWALD, 1997).

A teoria dos custos de transação busca explicar a organização econômica das empresas em termos dos custos de se gerenciar as interações entre as atividades econômicas, ou seja, determina o grau de verticalização e/ou desverticalização mais apropriado. 0 enfoque é na análise da escolha da forma de governança mais eficaz entre "hierarquia" e "mercado", considerando o oportunismo e a racionalidade limitada dos agentes (WILLIAMSON, 1985).

Na estrutura "hierárquica", a escolha está em produzir o bem ou serviço na própria empresa. Enquanto na estrutura por "mercado" a escolha é adquirir o bem ou serviço através de negociações com fornecedores independentes. No entanto, essa análise envolve um trade-off entre as economias providas do custo de produção ("hierarquia") e as economias no custo de transação ("mercado") (WILLIAMSON, 1981).

0 "mercado" coordena o fluxo através da oferta e da demanda entre diferentes empresas. Oferece certas vantagens em relação ao custo de produção, pois os fornecedores do "mercado" poderiam se beneficiar da combinação da demanda de diversos compradores para obter economias de escala e escopo. Por isso, geralmente, o "mercado" apresenta custo de produção mais baixo em comparação à estrutura "hierárquica". No entanto, o uso intensivo do "mercado" aumenta o custo de transação incorrido pelas empresas, deslocando as vantagens para a produção através da estrutura "hierárquica", isto é, na própria empresa (MILGROM; ROBERTS, 1992).

Entre os dois extremos ("mercado" e "hierarquia") existem formas intermediárias de governança denominadas "arranjos híbridos", que 
incluem as alianças estratégicas e joint ventures e as redes de empresas (WIGAND; PICOT; REICHWALD, 1997). Enquanto os "mercados" são caracterizados pela competição e a "hierarquia" pelo controle, as redes de empresa são definidas pelo relacionamento recíproco (POWELL, 1990).

As redes de empresas podem ser definidas como um arranjo interorganizacional, com empresas legalmente independentes, mas economicamente interdependentes que visam a cooperação em detrimento da concorrência (PFOHL; BUSE, 2000), para relações de troca, objetivos comuns ou complementares, laços comuns e relações sociais, que acabam por tornar-se contínuas ao longo do tempo (WILLIAMS, 2002).

A cooperação entre as empresas permite a combinação de competências essenciais e utilização de know-how de outras empresas, o compartilhamento de custos, riscos e conhecimentos para realizar pesquisas tecnológicas e explorar novas oportunidades de negócio, o oferecimento de uma linha de produtos de qualidade superior e mais diversificada, o compartilhamento de recursos subutilizados, o fortalecimento competitivo e do poder de compra para atuar no mercado internacional em favor do cliente (AMATO NETO, 2000).

Para havercooperaçãoénecessária a coordenação, pois os membros de uma rede têm comportamentos e prioridades diferentes, motivações e percepções dependentes do ambiente onde estão inseridos, podendo em algumas circunstâncias competir entre Si (CAMARINHA-MATOS; LIMA, 1999). As redes estão permanentemente no paradigma "cooperar ou competir", pois o desempenho, a competitividade e a posição perante o cliente necessitam da união de competências complementares que aproveitam o poder da cooperação com as vantagens competitivas (WILKINSON; YOUNG, 2002; LIPNACK; STAMPS, 1994). Nessa situação, a confiança é o principal fator nas relações entre empresas, capaz de tornar possivel que as estratégias empresariais se beneficiem do equilíbrio entre cooperação e competição. Ela é que faz com que os parceiros respeitem os compromissos assumidos entre as empresas pertencentes a determinada rede (AMATO NETO, 2000).

Com relação à direção, a cooperação pode acontecer na vertical ou na horizontal. A interdependência vertical surge na cooperação entre as empresas que pertencem a diferentes atividades da cadeia produtiva e se complementam entre si na produção ou comercialização do produto. Enquanto que na interdependência horizontal as relações de cooperação são entre empresas que produzem produtos similares no mesmo setor de atuação, e que buscam trocar conhecimentos ou recursos para desenvolver novos produtos e tecnologias (NASSIMBENI, 1998).

No desenvolvimento de sistemas, a cooperação entre as empresas, normalmente, ocorre na horizontal e propicia os efeitos da externalidade e das economias de escala e escopo (CLEMONS; ROW, 1992). As externalidades são os efeitos (positivos ou negativos) de uma determinada ação sobre terceiros não diretamente responsáveis por essa ação (FARINA; AZEVEDO; SAES, 1997). Nos sistemas de informação, a externalidade ocorre quando o valor do sistema para o cliente cresce na medida em que o número de seus participantes aumenta. As economias de escala surgem quando vários usuários que apresentam necessidades de utilizar sistemas de informação similares resolvem compartilhar os custos e riscos do desenvolvimento de suas aplicações (CLEMONS; ROW, 1992).

\section{Influência da Tl na redução das assimetrias de informação}

Na teoria econômica clássica supõe-se que todos os agentes possuem o mesmo nível de informação e que toda a informação é perfeita. Entretanto, essas considerações, em muitos casos, não correspondem à realidade, pois frequentemente o que se observa nos mercados são relacionamentos que envolvem assimetrias de informação entre os agentes econômicos, isto é, casos em que uma das partes envolvidas na transação tem alguma informação privada, não adquirível sem custos pela outra parte (AZEVEDO, 1997).

A partir da revisão dos conceitos da teoria clássica, surgiram teorias que contribuíram para analisar as situações que envolvem assimetria de informação. Uma delas é a teoria do agente e principal.

A teoria do agente e principal procura analisar os conflitos e custos resultantes de um relacionamento cooperativo onde a divisão de trabalho ocorre entre pessoas ou empresas com interesses divergentes (EISENHARDT, 1989).

Uma relação de agência é definida como:

[...] um contrato sob o qual uma ou mais pessoas (o principal) contrata outra pessoa (o agente) para realizar algum serviço em seu interesse no qual envolve delegação de alguma autoridade para tomada de decisão para o agente. Se ambas as partes da relação são maximizadoras de utilidade, existe uma boa razão para acreditar que o agente não atuará sempre nos melhores interesses do principal (JENSEN; MECKLING, 1976, p. 5). 
Assim, os problemas da relação entre agente e principal surgem quando os objetivos dos dois são conflitantes e nos casos em que é difícil ou custoso para o principal verificar as ações do agente, pois nesses casos o agente poderá negligenciar o cumprimento de suas tarefas, prejudicando os objetivos do principal (EISENHARDT, 1989).

Há dois aspectos da teoria do agente e principal, causados pela assimetria da informação: a seleção adversa e o risco moral.

Na seleção adversa, a assimetria de informação ocorre devido à falta de informações por parte do principal acerca das habilidades dos agentes do mercado (AKERLOF, 1970). Nessa situação, o agente pode ocultar informações que não sejam interessantes para aquele bem transacionado, atuando de forma oportunista (VARIAN, 2000).

Uma alternativa para esse tipo de comportamento é criar mecanismos de sinalização no mercado. Nesse caso, os agentes de qualidade agiriam de modo a fornecer informações confiáveis acerca do bem transacionado (SPENCE, 1973). 0 principal poderia exigir, por exemplo, certificados e garantias de longo prazo para distinguir a qualidade dos agentes (WIGAND; PICOT; REICHWALD, 1997; PINDYCK; RUBINFELD, 2002).

Já o risco moral ocorre após o estabelecimento de contrato entre o agente e o principal, caso o agente não respeite os termos contratuais, agindo de forma oportunista (PONDÉ, 2002), de acordo com seus interesses para obter vantagens em detrimento da outra parte (AZEVEDO, 1997). 0 risco moral também pode estar relacionado a situações de ação oculta (VARIAN, 2000), em que o agente age de forma oportunista sem o conhecimento do principal.

Gurbaxani e Whang (1991) afirmam que sob a perspectiva da teoria do agente e principal, a disponibilidade de dispositivos de monitoramento que sejam acessíveis e efetivos é um fator decisivo para reduzir os problemas da assimetria de informação, pois os sistemas de informação contribuem para essa finalidade provendo ferramentas efetivas para o monitoramento das ações dos agentes e registrando o histórico de desempenho do agente.

Ao informar o principal sobre as ações executadas pelo agente, os sistemas de informação tendem a inibir a conduta oportunista do agente, uma vez que ele tem a percepção de que dificilmente pode ludibriar o principal (EISENHARDT, 1989; VAN DER HEIJDEN et al., 1995).

Clemons e Row (1992) também acreditam que Tl diminui os riscos da transação, pois o uso de canais de informação permite o monitoramento mais detalhado do desempenho das empresas, consequentemente diminui-se a chance de as empresas se desviarem dos objetivos coletivos para um comportamento individual e oportunista.

Nos mercados eletrônicos a assimetria de informação não pode ser evitada completamente, contudo o fácil acesso à informação permite às empresas diminuírem seus custos de agenciamento ao reduzir os custos de aquisição e de análise da informação (ALBERTIN, 2004).

\section{Metodologia de pesquisa}

Após apresentar a revisão bibliográfica sobre os elementos conceituais, este artigo propõe uma pesquisa empírica através de um estudo de caso de caráter exploratório. 0 estudo de caso abordado tem as seguintes características: 1) busca responder como e por que o desenvolvimento do e-marketplace é influenciado pelas teorias de redes de cooperação e de assimetrias de informação; 2) os eventos comportamentais da rede de cooperação não podem ser controlados; e 3) tem enfoque em eventos contemporâneos (YIN, 2001).

Inicialmente, foram coletados os dados para entendimento do negócio. 0 levantamento dos dados, cuja fonte de informações baseou-se principalmente em documentos administrativos e entrevistas com os envolvidos, buscou examinar a organização e suas exigências a partir de cinco perspectivas inter-relacionadas: objetivos, regras de negócio, processos de negócio, atores e requisitos do sistema de informação. As questões consideradas no levantamento dos dados são apresentadas no Quadro 1 (BUBENKO; PERSSON; STIRNA, 2001).

A partir dos dados coletados foram desenvolvidos os modelos conceituais utilizando EKD (Enterprise Knowledge Development). O EKD fornece uma forma sistemática e controlada de analisar, entender e documentar o papel desempenhado pelos sistemas de informação nos objetivos estratégicos da organização (BUBENKO; PERSSON; STIRNA, 2001). Deve-se ressaltar que o termo "organização" expressa uma empresa ou conjunto de empresas no qual se deseja aplicar as técnicas de modelagem empresarial.

Apesar de a modelagem EKD ser composta por sete tipos de modelo - modelo de objetivos, de regras de negócio, de processos de negócio, de atores, de conceitos e de requisitos do sistema de informação (BUBENKO; PERSSON; STIRNA, 2001) -, restringiu-se o objeto de estudo aos dois modelos mais relevantes para o propósito do artigo: 
Quadro 1. Levantamento dos dados.

\begin{tabular}{|c|l|}
\hline \multirow{2}{*}{ Objetivos } & Quais são as estratégias da organização? \\
& Quais objetivos a organização deseja alcançar? \\
& Como esse objetivo pode ser alcançado? \\
& O objetivo pode ser definido operacionalmente, decompondo-o em sub-objetivos de apoio? \\
& Existe algum problema impedindo o objetivo? \\
& Qual é a causa do problema? \\
& Existem oportunidades que poderiam ser vislumbradas? \\
Quais ações poderiam ser tomadas para melhorar a situação?
\end{tabular}

- Modelo de objetivos: Representa as metas que a organização pretende alcançar ou evitar.

- Modelo de requisitos do sistema de informação: Descreve os requisitos para os sistemas computadorizados que suportem os objetivos e processos de negócio da organização.

\section{Análise de um estudo de caso na construção civil}

No Brasil, o setor da construção civil desempenha importância histórica na economia nacional. 0 setor apresenta elevada participação na geração do Produto Interno Bruto (PIB) e representa 7,7\% das ocupações do mercado de trabalho (INSTITUTO..., 2004).

Tanto no caso do segmento de edificação industrial quanto no caso do subsetor de construção pesada é comum as obras serem gerenciadas por meio da figura jurídica do "consórcio". Os consórcios de empresas são grupos ou reorganizações entre quaisquer companhias ou sociedades unidas para a execução de um empreendimento sem, todavia, estabelecer um vínculo na forma de uma fusão ou incorporação, ou pela criação de uma sociedade, que no caso brasileiro possui legislação própria que define as relações entre as empresas na Lei das Sociedades Anônimas como um contrato (LEÃO, 2000).
0 estabelecimento de parcerias permite às construtoras economizarem recursos e ao mesmo tempo aumenta a competitividade através da troca de experiências entre as equipes e da elaboração de projetos com melhor qualidade (BALDWIN; THORPE; CARTER, 1999).

No Brasil, para cada consórcio criado, quase nenhum recurso é aproveitado de projetos anteriores, sendo que atividades como gestão de recursos humanos, contas a pagar e a receber, controle de insumos e compras poderiam ser estrategicamente reaproveitadas. Estima-se que atividades colaborativas no setor da construção civil podem gerar expressivas reduções de custos, na ordem de 5 a 10\% para cada novo consórcio criado (EBUSINESS BRASIL, 2004b).

Além da colaboração, outro fator que permite aumentar a competitividade no setor da construção civil é a aplicação de $\mathrm{Tl}$ nos processos de negócio (ZARL1, 2004). Apesar disso, o setor da construção civil ainda é considerado atrasado no uso da Tl, em comparação com outros setores, devido às barreiras culturais e às deficiências na padronização da tecnologia (NASCIMENTO; SANTOS, 2003). Porém, o setor está evoluindo, principalmente após os avanços da internet.

Há alguns anos, as soluções tecnológicas para integração de dados entre construtoras e seus 
fornecedores eram limitadas e caras. 0 EDI (Eletronic Data Interchange), que desempenhou relevante papel como sistema de integração em alguns setores industriais (montadoras de automóveis, bancos e grandes varejistas), teve baixo impacto na indústria da construção, pois o pequeno volume de transações tornava inviável os gastos com licenças e contratação das redes privadas de mensagens (JACOSKI; LAMBERTS, 2002).

Assim, por um determinado período, o uso de $\mathrm{Tl}$ nas relações externas com clientes e fornecedores permanecia sem grandes inovações devido aos altos custos de investimento necessários para automatizar essas atividades. Somente a partir da difusão da internet como ambiente transacional ocorrido na segunda metade da década de 90 é que se tornou possivel a expansão de tecnologias que melhoraram a interação entre as empresas (FLEURY, 2001).

Tendo em vista as oportunidades geradas pela colaboração e pela internet, sete grandes construtoras brasileiras, juntamente com a Associação Brasileira de E-business, formaram o Comitê Setorial da Construção. Caracterizado como uma rede de cooperação entre construtoras, ele tem como missão formular propostas e definir padrões de tecnologias que estimulem as práticas de colaboração no setor da construção civil (EBUSINESS BRASIL, 2004a).

Para descrever os objetivos do comitê e as questões associadas para atingi-los foi desenvolvido o modelo de objetivos (Figura 1). 0 modelo de objetivos serve como importante meio para esclarecer questões sobre os rumos que o comitê deve seguir, definir quais objetivos são prioritários e determinar qual o relacionamento entre os objetivos e os problemas ocultos na realização das metas (BUBENKO; PERSSON; STIRNA, 2001).

Em princípio, o enfoque na formação do Comitê Setorial da Construção (objetivo 6) é promover a integração entre construtoras e fornecedores (objetivo 5), para melhorar a aquisição de produtos e serviços pelas construtoras (objetivo 1).

Com o intuito de obter mais clareza sobre o objetivo 1 utilizou-se a técnica de refinamento com o relacionamento "AND" (BUBENKO; PERSSON; STIRNA, 2001) para representar que os sub-objetivos objetivo 1.1 e objetivo 1.2 devem ser atendidos simultaneamente para satisfazer o objetivo original.

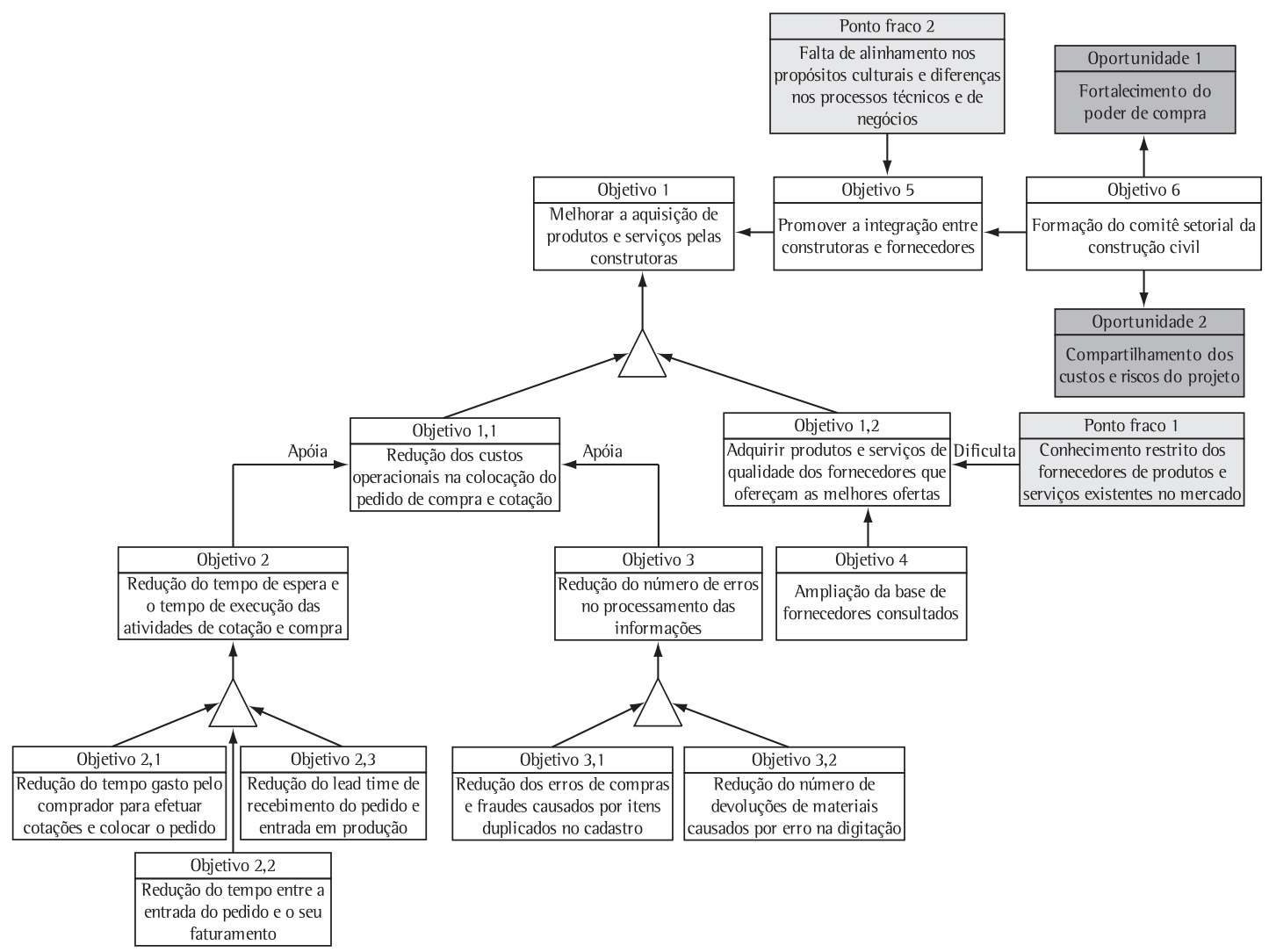

Figura 1. Modelo de objetivos. 
O objetivo 1.1 tem a finalidade de reduzir custos operacionais na colocação do pedido de compra e cotação pelas construtoras. Esse objetivo pode ser operacionalizado através da redução do tempo (objetivo 2) e do número de erros (objetivo 3) no processo de aquisição de produtos e serviços.

O objetivo 1.2 visa adquirir produtos e serviços de qualidade dos fornecedores que ofereçam as melhores ofertas, porém o conhecimento restrito existente sobre eles no mercado (ponto fraco 1) limita o número de fornecedores a serem considerados. Uma das formas de solucionar o problema é ampliar a base de fornecedores consultados (objetivo 4).

Tanto o objetivo 1.1 quanto o objetivo 1.2 podem ser vistos como diferentes formas para redução dos custos de transação. No objetivo 1.1, o foco é tornar mais eficiente o processo de aquisição de bens e serviços e consequentemente diminuir os custos de coordenação com o mercado. Já no objetivo 1.2, em que o interesse é encontrar fornecedores qualificados que atendam às necessidades demandadas, o relacionamento entre as empresas pode estar sujeito aos problemas da seleção adversa, se acaso a construtora (principal) não tiver informações suficientes acerca da qualidade dos fornecedores (agente) no momento de sua contratação. Portanto, também deve-se buscar meios que reduzam os custos decorrentes das assimetrias de informação.

Para auxiliar as construtoras no processo de compra e cotação e promover a integração entre as construtoras e fornecedores foi necessário desenvolver um e-marketplace vertical (atende o setor específico da construção civil), cujos requisitos são apresentados no modelo de requisitos do sistema de informação (Figura 2).

No intuito de definir os requisitos iniciais do e-marketplace, algumas funcionalidades consideradas prioritárias para as construtoras foram imediatamente propostas, entre elas 0 compartilhamento dos cadastros de fornecedores e de materiais.

0 cadastro de fornecedores (requisito SI 1) contém os dados dos fornecedores e sua respectiva categoria de fornecimento. Esses dados serão disponibilizados para consultas entre as construtoras do grupo, auxiliando a ampliação da base de fornecedores consultados (objetivo 4) no processo de seleção

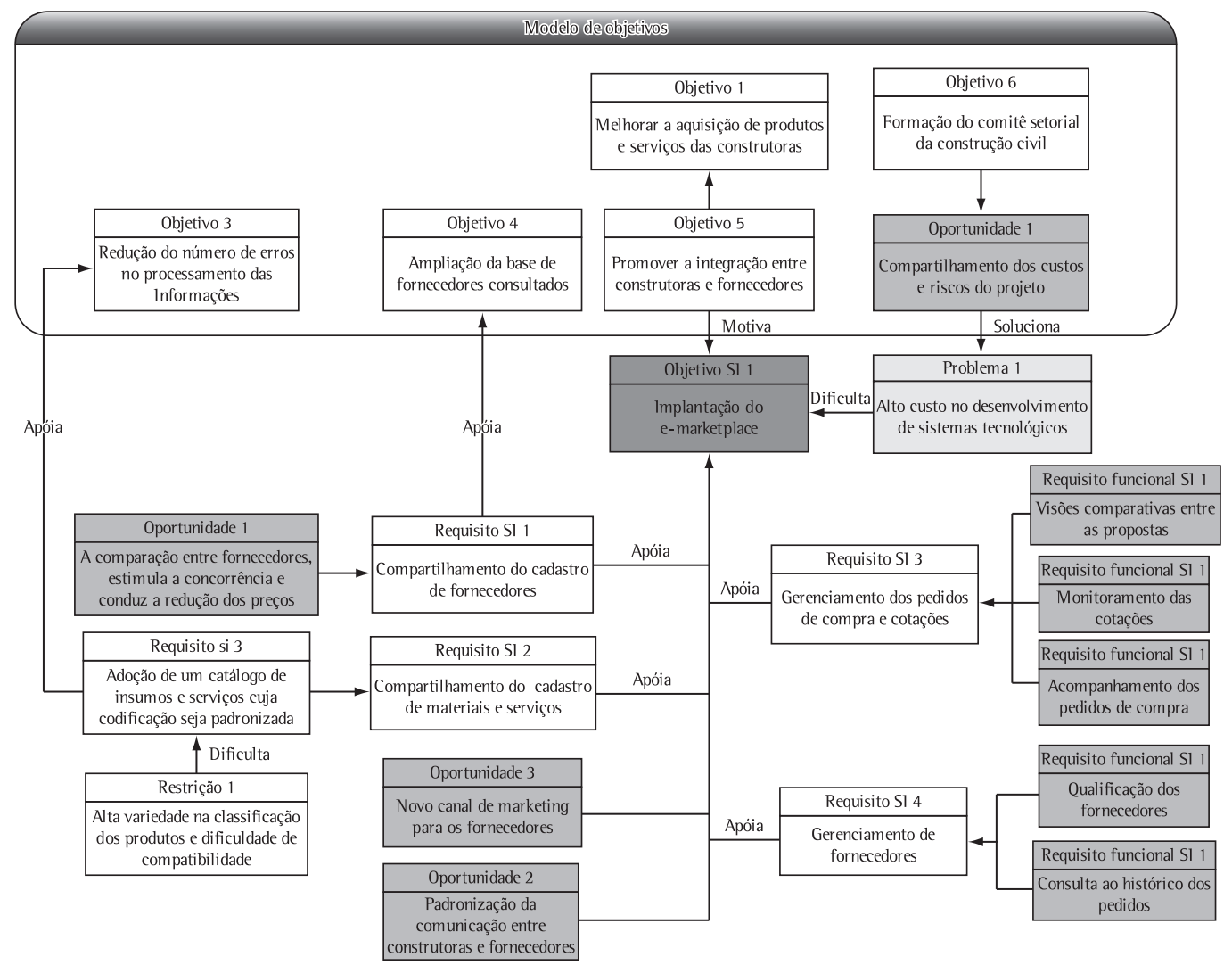

Figura 2. Modelo de requisitos técnicos - Comitê Executivo de Construção Civil. 
destes. Uma vantagem decorrente desse requisito é que o aumento no número de comparações estimula a concorrência e leva à redução dos preços (oportunidade 1). Para os fornecedores a vantagem é que, uma vez cadastrados no sistema, seus dados ficarão disponíveis para todas as construtoras do grupo servindo como canal de marketing adicional (oportunidade 2).

No cadastro de materiais e serviços (requisito S1 2), os itens são classificados de acordo com um padrão de codificação (requisito Sl 5). Essa codificação é definida conjuntamente entre as construtoras para utilizar com seus fornecedores. A vantagem da padronização é que a comunicação entre as empresas torna-se mais clara e simples, evitando ambiguidades na especificação dos itens (nomenclatura, unidades de medida e classificação) e, portanto, reduzindo o número de erros no processamento das informações (objetivo 3). Todavia, sua adoção pode se tornar complexa por causa da alta variedade na classificação dos materiais e da dificuldade de compatibilidade entre as construtoras (restrição 1).

Frente à discussão sobre os requisitos para realização das atividades de cotação e compras foram propostos as funcionalidades de gerenciamento das atividades de compra e cotação (requisito Sl 3) e o gerenciamento de fornecedores (requisito Sl 4). Ambos buscam elevar a capacidade das empresas em planejar, negociar e tomar uma decisão de compra mais eficiente, de modo a reduzir os custos de transação e as assimetrias de informação no processo de seleção dos fornecedores.

Nessa seleção, a funcionalidade de mapas comparativos permite à construtora comparar as propostas apresentadas pelos fornecedores, em que os melhores preços ficam destacados. Deve-se observar que o custo é importante fator na escolha do fornecedor, no entanto basear a decisão de compra unicamente nesse critério pode ser uma estratégia arriscada, pois para alguns tipos de insumos, ao optar por pagar um valor baixo, o comprador tende a selecionar somente os fornecedores de baixa qualidade, ocasionando problemas relacionados à seleção adversa. Para evitar isso, é necessário que as construtoras analisem a qualificação dos fornecedores a partir do histórico de transações, considerando também critérios como qualidade, confiabilidade e honestidade.

Para prover informações sobre a qualificação dos fornecedores, as construtoras poderão atribuir notas de avaliação para o produto ou serviço recebido. Essa qualificação será compartilhada entre as construtoras, permitindo que a avaliação sobre a performance dos fornecedores esteja disponível para o grupo.

Essa avaliação é importante, pois sem ela alguns deles não teriam incentivos em fornecer seus produtos e serviços com qualidade. E a atitude de negligência em relação ao fornecimento pode causar os problemas de assimetria de informação (MILGROM; ROBERTS, 1992).

Nas transações comerciais entre empresas, uma delas pode incorrer na seleção adversa se no processo de seleção de fornecedor houver a contratação de uma empresa para fornecer um insumo cujas especificações ela própria já saiba que não terá capacidade de atender. Por outro lado, o risco moral ocorrerá se uma empresa fornecedora inicialmente produzir um insumo num nível de qualidade aceitável e, após estabelecer o contrato, reduzir o nível de qualidade do insumo com o objetivo de diminuir custos (FIANI, 2002).

Segundo Milgrom e Roberts (1992), quando os compradores não conseguem monitorar a qualidade dos bens ou serviços que recebem, os fornecedores poderão negligenciar suas atividades, substituir 0 fornecimento por produtos de baixa qualidade ou empregar pouco esforço e dedicação na realização dos serviços. Assim, há a tendência de determinados fornecedores diminuírem a qualidade de seus produtos e serviços para aumentar os lucros até que os consumidores percebam a mudança.

Uma alternativa para reduzir os problemas decorrentes da assimetria de informação é monitorar frequentemente a qualidade e a reputação dos fornecedores em transações repetidas no passado e comunicar essas informações o mais rápido possível aos demais compradores do grupo, evitando a contratação de fornecedores que não apresentem desempenho satisfatório (TIROLE, 1997). Os sistemas de informação têm papel importante nesse processo, pois viabilizam a infraestrutura tecnológica necessária para a disseminação rápida da informação sobre a reputação dos agentes entre os compradores (EISENHARDT, 1989).

A combinação entre a cooperação das construtoras com as funcionalidades do e-marketplace permite minimizar os problemas da seleção adversa, visto que, mesmo numa situação em que a construtora nunca tenha contratado um determinado fornecedor, ela terá como determinar a qualidade dele a partir das informações compartilhadas com outras construtoras do grupo.

A reputação é um importante mecanismo para assegurar que os contratos sejam honrados, pois, embora leve tempo para ser consolidada, pode ser destruída rapidamente (MILGROM; ROBERTS, 
1992; GULATI; NOHRIA; ZAHEER, 2000). Nas redes de empresa, o efeito da reputação torna o comportamento oportunista mais custoso porque, ao agir de modo antiético, não cumprindo com sua responsabilidade frente aos contratos já firmados, a empresa danifica sua própria imagem, influenciando negativamente seu relacionamento com os parceiros atuais e potenciais (ALSTYNE, 1996; GULATI; NOHRIA; ZAHEER, 2000). Assim, pelo fato de uma atitude oportunista do fornecedor poder resultar em uma eventual interrupção de transações futuras com demais integrantes da rede, os próprios fornecedores hesitariam em fazer a mesma coisa contra uma das construtoras, minimizando o risco moral.

Contudo, há o risco de que as notas atribuídas aos fornecedores induzam a formação de grupos e as preferências perigosas, podendo prejudicar bons fornecedores ou mesmo elevar os péssimos. Assim, as informações sobre a qualificação dos fornecedores são válidas somente se as construtoras qualificá-los de modo imparcial. Apesar disso, a distorção da informação pode ser minimizada, uma vez que a qualificação do fornecedor é compartilhada, feita a partir de uma ponderação entre as avaliações realizadas pelas construtoras.

Em relação às barreiras para criação do e-marketplace, constatou-se que o quesito tecnológico não é o problema. 0 principal impedimento seria a falta de alinhamento nos propósitos culturais e as diferenças nos processos de negócios. Para minimizar, foi proposto que a integração seja baseada em um plano estratégico que considere a integração dos fornecedores a partir do relacionamento de cada construtora. De acordo com os integrantes, a solução somente terá efetividade se utilizada por grande parte das construtoras, evitando que o fornecedor tenha que adequá-la a diferentes tipos de processos de compras para cada construtora.

Outro problema identificado são os custos envolvidos no desenvolvimento do sistema. Contudo, isso pode ser minimizado, uma vez que os custos e riscos dessa etapa serão compartilhados pelas construtoras. Além disso, a cooperação propicia vantagens relacionadas à economia de escala e escopo, pois a definição de um padrão tecnológico permite às empresas reaproveitar os recursos em projetos futuros e também elimina os custos de investimentos redundantes que ocorreriam se cada empresa desenvolvesse sistemas proprietários similares (CLEMONS; ROW, 1992).

\section{Considerações finais}

A percepção de que a cooperação propicia vantagens, que dificilmente seriam alcançadas isoladamente, tem levado à formação das redes de cooperação para o desenvolvimento de e-marketplaces verticais. De modo geral, os fatores determinantes para a colaboração no desenvolvimento dos e-marketplaces têm sido a possibilidade de compartilhamento de custos e riscos, os efeitos positivos das externalidade e das economias de escala, a maior influência sobre o mercado e o fortalecimento do poder de compra.

Sob a perspectiva da teoria dos custos de transação, os mercado eletrônicos propiciam a redução dos custos de coordenação com o mercado através da intermediação eletrônica, conduzindo ao uso intensivo do mercado - em contraposição à diminuição das estruturas hierárquicas - como forma de coordenar as atividades econômicas (MALONE; YATES; BENJAMIN, 1987).

Embora tradicionalmente os e-marketplaces sejam analisados como uma forma de redução dos custos de coordenação com o mercado, também é importante analisar seus efeitos sobre as assimetrias de informação. As teorias relacionadas com assimetrias de informação orientam $\mathrm{n}$ o sentido de minimizar as chances de que interesses individuais se sobreponham aos coletivos numa relação sujeita à distribuição irregular de informação. Uma das formas que a literatura sugere para controlar o comportamento oportunista do agente é por meio do monitoramento das transações repetidas no passado (TIROLE, 1997) com a utilização de sistemas de informação (EISENHARDT, 1989).

Para uma maior compreensão do assunto, foi realizado um estudo de caso no setor da construção civil em que se analisou o Comitê Setorial da Construção. 0 comitê é uma iniciativa que demonstra o interesse das construtoras brasileiras em melhorar a competitividade do setor através da cooperação e da utilização da Tl.

No estudo de caso foram analisados como e por que as construtoras estão cooperando para a construção de um e-marketplace. $\mathrm{Na}$ análise do processo de aquisição de produtos e serviços constatou-se - através de modelo de objetivos e modelo de componentes e requisitos técnicos que o compartilhamento das informações sobre transações passadas permite que os integrantes da rede possam avaliar a qualidade dos fornecedores, reduzindo as chances da seleção adversa. E que a própria formação da rede de cooperação pode inibir o risco moral dos fornecedores, visto que um comportamento oportunista poderia prejudicar sua reputação perante os outros membros da rede. Consequentemente, a redução das assimetrias de informação entre fornecedores e construtoras pode fortalecer o poder de compra, propiciando ganhos coletivos para as construtoras. 
Outro aspecto importante da pesquisa foi a estruturação sobre o conhecimento do projeto através dos modelos conceituais em EKD. A vantagem do EKD é que a definição dos modelos de objetivos, processos de negócio e requisitos técnicos permite analisar o alinhamento entre o planejamento estratégico e as funcionalidades do sistema de informação (BUBENKO; PERSSON; STIRNA, 1998). Os modelos serviram como referência para as discussões do comitê, sendo utilizados como base para os requisitos do sistema de informação.

\section{Referências}

AKERLOF, G. A. The market for 'lemons': quality uncertainty and the market mechanism. Quarterly Journal of Economics, v. 84, n. 3, p. 488-500, 1970.

ALBERTIN, A. L. Comércio eletrônico: modelos, aspectos e contribuições de sua aplicação. São Paulo: Atlas, 2004.

ALSTYNE, M. V. The state of network organization: a survey in three frameworks. Working Paper Series 192, MIT Center for Coordination Science, 1996.

AMATO NETO, J. Redes de cooperação produtiva e clusters regionais: oportunidades para as pequenas e médias empresas. São Paulo: Atlas, 2000.

AZEVEDO, P. F. A nova economia institucional. In: FARINA, E. M. M. Q.; AZEVEDO, P. F.; SAES, M. S. M. Competitividade: mercado, estado e organizações. São Paulo: Singular, 1997. p. 29-109.

BAKOS, Y. The emerging role of electronic marketplaces on the internet. Communications of the ACM, v. 41, n. 8 , p. 35-42, 1998.

BALDWIN, A. N.; THORPE, A.; CARTER, C. The use of electronic information exchange on construction alliance projects. Automation in construction, v. 8, p. 651-662, 1999.

BUBENKO, J.; PERSSON, A.; STIRNA, J. EKD User Guide. Stockholm, Sweden, 2001. Disponivel em: <http://www. verbundplan.at/HyperKnowledge/pdf/D3_appb_final_ version_2001_10_31.pdf>. Acesso em: 17 mar. 2005.

BYRNS, R. T.; STONE, G. W. Comportamento estratégico. In: BYRNS, R. T.; STONE, G. W. Microeconomia. São Paulo: Makron, 1996. p. 262-275.

CAMARINHA-MATOS, L. M.; LIMA, C. P. Coordination and configuration requirements in a virtual enterprise. In: WORKING CONFERENCE ON INFRASTRUCTURE FOR VIRTUAL ENTERPRISE - PRO-EV'99, 1999, Porto, Portugal. Proceedings... p. 49-64.

CLEMONS, E. K.; ROW M. C. Information technology and industrial cooperation: the changing economics of coordination and ownership. Journal of Management Information Systems, v. 9, n. 2, p. 9-28, 1992.

COASE, R. H. The nature of the firm. Economica, v. 4, p. 386-405, 1937.

EBUSINESS BRASIL. Atas de reunião do Comitê executivo da construção civil. Acesso em: 01 dez. 2004a.

EBUSINESS BRASIL. Colaboração é pauta no setor de construção. Disponível em: <http://www.ebusinessbrasil. com.br/txt_perfil_pain.php?id=318>. Acesso em: 03 ago. 2004b.
EISENHARDT, K. M. Agency theory: na assessment and review. The Academy of Management Review, v. 14, n. 1, p. 57-74, 1989.

FARINA, E. M. M. Q.; AZEVEDO, P. F.; SAES, M. S. M. Competitividade: mercado, estado e organizações. São Paulo: Singular, 1997.

FIANI, R. Teoria dos custos de transação. In: KUPFER, D.; HASENCLEVER, L. (Org.). Economia industrial: fundamentos teóricos e práticos no Brasil. Rio de Janeiro: Campus, 2002. p. 267-286.

FLEURY, A. L. Dinâmicas organizacionais em mercados eletrônicos. São Paulo: Atlas, 2001.

GEBREKIDAN, D. A.; AWUAH, G. B. Interorganizational cooperation: a new view of strategic alliances: the case of Swedish firms in the international market. Industrial Marketing Management, n. 31, p. 679-693, 2002.

GULATI, R.; NOHRIA, N.; ZAHEER, A. Strategic networks. Strategic Management Journal, v. 21, p. 203-215, 2000.

GURBAXANI, V.; WHANG, S. The impact of Information Systems on Organizations and Markets. Communications of the ACM, p. 59-73, 1991.

INSTITUTO BRASILEIRO DE GEOGRAFIA E ESTATÍSTICA - IBGE. Pesquisa Mensal de Emprego. Disponível em: <http://www.ibge.gov.br/home/ presidencia/ noticias/27042004pmehtml.shtm>. Acesso em: 23 jul. 2004.

JACOSKI, C. A.; LAMBERTS, R. Vetores de virtualização da indústria da construção: $A$ integração da informação como elemento fundamental ao uso de Tl. In: ENCONTRO NACIONAL DE TECNOLOGIA DO AMBIENTE CONSTRUIDO - ENTAC, 2002. Anais...

JENSEN, M. C.; MECKLING, W. Theory of the firm: managerial behavior, agency costs and ownership structure. Journal of Financial Economics, v. 3, n. 4, p. 305-360, 1976.

KANDAMPULLY, J. B2B relationships and networks in the internet age. Management Decision, v. 41, n. 5, p. 443-451, 2003.

KAPLAN, S.; SAWHNEY, M. E-hubs: the new b2b marketplaces. Harvard Business Review, v. 78, n. 3, p. 97-103, 2000.

LEÃO, L. C. G. Uma contribuição ao planejamento de consórcio de empresas. Dissertação (Mestrado)-Faculdade de Economia, Administração e Contabilidade, Universidade de São Paulo, São Paulo, 2000.

LIPNACK, J.; STAMPS, J. Rede de informações. São Paulo: Makron Books, 1994.

MALONE, T. W.; YATES, J.; BENJAMIN, R. l. Eletronic markets and eletronic hierarchies. Communications of the ACM, v. 30, n. $6,1987$.

MILGROM, P.; ROBERTS, J. Economics, organization and management. New Jersey: Prentice-Hall, 1992.

NASCIMENTO, L. A.; SANTOS, E. T. A indústria da construção na era da informação. Revista Ambiente Construído, v. 3 , n. 1, p. 69-81, 2003.

NASSIMBENI, G. Network structures and co-ordination mechanisms a taxonomy. International Journal of Operations \& Production Management, v. 18, n. 6, p. 538-554, 1998.

PFOHL, H.; BUSE, H. P. Inter-organizational logistics systems in flexible production networks. An organizational capabilities perspective. International Journal of Physical Distribution \& Logistics Management, v. 30, n. 5, p. 388-408, 2000. 
PINDYCK, R. S.; RUBINFELD, D. L. Mercados com informação assimétrica. In: PINDYCK, R. S.; RUBINFELD, D. L Microeconomia. São Paulo: Makron, 2002. p. 667-700.

PONDÉ, J. L. Organização das grandes corporações. In: KUPFER, D.; HASENCLEVER, L. (Org.). Economia industrial: fundamentos teóricos e práticos no Brasil. Rio de Janeiro: Campus, 2002. p. 287-306.

POWELL, W. W. Neither market nor hierarchy: network forms of organization. Research in Organization Behavior, v. 12, p. 295-336, 1990.

SPENCE, M. Job market signaling. Quarterly Journal of Economics, v. 87, p. 355-374, 1973.

TIROLE, J. Product selection, quality and advertising. In: TIROLE, J. The theory of industrial organization. Massachusetts: MIT Press, 1997. p. 95-129.

VAN DER HEIJDEN, $\mathrm{H}$. et al. Redesigning process control mechanisms using EDl. An agency-theoretic perspective. In: INTERNATIONAL CONFERENCE ON SYSTEM SCIENCES, 28., Hawaii, 1995. Proceedings... p. 388-397.

VARIAN, H. R. Informação assimétrica. In: VARIAN, H. R. Microeconomia: princípios básicos. São Paulo: Campus, 2002. p. 716-739.

WIGAND, R. T.; BENJAMIN, R. I. Electronic commerce: effects on electronic markets. Journal of Computer-Mediated Communication, 1995. Disponível em: <http://www. ascusc.org/jcmc/vol1/issue3/wigand.html> Acesso em: 16 ago. 2004.

WIGAND, R.; PICOT, A.; REICHWALD, R. Information, organization and management. expanding markets and corporate boundaries. Chichester: Wiley, 1997.

WILKINSON, 1; YOUNG, L. On cooperating Firms, relations and networks. Journal of Business Research, n. 55, p. 123-132, 2002.

WILLIAMS, T. Cooperation by design: structure and cooperation in interorganizational networks. Journal of Business Research, v. 5867, p. 1-9, 2002.

WILLIAMSON, 0. E. The economic institutions of capitalism: firms, markets, relational contracting. New York: The Free Press, 1985.

WILLIAMSON, 0. E. The economics of organization: the transaction cost approach. The American Journal of Sociology, v. 87, n. 3, p. 548-577, 1981.

YIN, R. K. Estudo de caso: planejamento e métodos. São Paulo: Bookman, 2001.

ZARLl, A. Innovation co-ordination, transfer and deployment through networked co-operation in the construction industry. $\mathrm{ICCl}$ Final Report. Disponivel em: <http://cic. vtt.fi/projects/icci/deliverables/icci_final_public_report. pdf> Acesso em: 30 jul. 2004.

\title{
Formation of inter-organizational cooperation network to vertical e-marketplace development
}

\begin{abstract}
Nowadays, vertical e-marketplace projects are coordinated by companies of the same economic sector, sometimes competitors, which get together to increase the competitiveness of the sector. In this context, this paper analyzes determinant factors for cooperation in e-marketplace development and their effects on asymmetric information between buyers and sellers. Finally, a case study in the civil construction sector is presented, where a few huge Brazilian construction companies have proposed to share costs and risks to e-marketplace development.
\end{abstract}

Keywords

Cooperation network. E-commerce. Asymmetric information. Civil construction. 